\title{
AMENABILITY AND THE STRUCTURE OF THE ALGEBRAS $A_{p}(G)$
}

\author{
BRIAN FORREST
}

\begin{abstract}
A number of characterizations are given of the class of amenable locally compact groups in terms of the ideal structure of the algebras $A_{p}(G)$. An almost connected group is amenable if and only if for some $1<p<\infty$ and some closed ideal $I$ of $A_{p}(G), I$ has a bounded approximate identity. Furthermore, $G$ is amenable if and only if every derivation of $A_{p}(G)$ into a Banach $A_{p}(G)$-bimodule is continuous.
\end{abstract}

\section{INTRODUCTION}

Let $G$ be a locally compact group. In [7] Eymard defined the Fourier algebra $A(G)$ of $G$ to be the linear subspace of $C_{0}(G)$ consisting of all functions of the form $u(x)=(f * \tilde{g})^{\vee}(x)$, where $f, g \in L_{2}(G), f^{\vee}(x)=f\left(x^{-1}\right)$ and $\tilde{f}(x)=\overline{f\left(x^{-1}\right)}$. The space $A(G)$ can be identified with a quotient space of the projective tensor product $L_{2}(G) \otimes_{\gamma} L_{2}(G)$. With respect to pointwise multiplication and the quotient norm, $A(G)$ is a commutative Banach algebra.

In [15], Herz introduced the $L_{p}$-versions of Eymard's algebra. He defined $A_{p}(G)$ to be the space of functions of the form $u(x)=\sum_{n=1}^{\infty}\left(f_{i} * \tilde{g}_{i}\right)^{\vee}$ where $f_{i} \in L_{p}(G), g_{i} \in L_{q}(G), 1 / p+1 / q=1,1<p<\infty$, and $\sum_{n=1}^{\infty}\left\|f_{i}\right\|_{p}\left\|g_{i}\right\|_{q}<$ $\infty$. Then

$$
\|u\|_{A_{p}(G)}=\inf \left\{\sum_{n=1}^{\infty}\left\|f_{i}\right\|_{p}\left\|g_{i}\right\|_{q} \mid \sum_{n=1}^{\infty}\left(f_{i} * \bar{g}_{i}\right)^{\vee}\right\}
$$

determines a norm on $A_{p}(G)$ with respect to which $A_{p}(G)$ is a Banach algebra. When $p=2, A_{p}(G)=A(G)$.

We began a study of the structure of the closed ideals of $A(G)$ in [9 and 10]. The present investigation will extend many of the principal results of these earlier works to the $A_{p}(G)$ algebras. We will also prove a number of results which are new even for $p=2$. In particular, we show that if $G$ is almost connected, then $G$ is amenable if and only if some closed ideal $I$ in $A_{p}(G)$ has a bounded approximate identity for some $1<p<\infty$. For the class of amenable

Received by the editors July 9, 1990 and, in revised form, July 15, 1992.

1991 Mathematics Subject Classification. Primary 43A07, 43A15; Secondary 46J10.

Key words and phrases. Amenable groups, Herz algebra, bounded approximate identities, ideal, automatic continuity, derivations. 
groups we are able to improve considerably the main result of [6] which is concerned with invariant projections on $A_{p}(G)$-submodules of $\operatorname{PM}_{p}(G)$.

\section{Preliminaries}

Throughout this paper, $G$ will denote a locally compact group with a fixed left Haar measure $\lambda_{G}$. For $1<p<\infty, A_{p}(G)$ will denote the Banach algebra of continuous functions defined in $\S 1$.

Denote by $\operatorname{PF}_{p}(G)$ the closure of $L^{1}(G)$, considered as convolution operators on $L_{p}(G)$, with respect to the norm topology of $\mathscr{B}\left(L_{p}(G)\right)$, the bounded linear operators on $L_{p}(G)$. The weak operator topology closure of $L^{1}(G)$ is denoted by $\operatorname{PM}_{p}(G)$. The spaces $\operatorname{PF}_{p}(G)$ and $\operatorname{PM}_{p}(G)$ are referred to as the $p$-pseudofunctions and the $p$-pseudomeasures respectively. It can be shown that $\mathrm{PM}_{p}(G)=A_{p}(G)^{*}$. When $p=2, \mathrm{PM}_{2}(G)$ is usually denoted by $V N(G)$ while $\mathrm{PF}_{2}(G)$ is $C_{r}^{*}(G)$, the reduced group $C^{*}$-algebra of $G$ (see [7]).

$B_{p}(G)$ is the multiplier algebra of $A_{p}(G)$, consisting of the continuous complex-valued functions $v$ on $G$ such that $v u \in A_{p}(G)$ for every $u \in A_{p}(G)$. Define a norm on $B_{p}$ by

$$
\|v\|_{B_{p}(G)}=\left\{\|u v\|_{A_{p}(G)} \mid u \in A_{p}(G) \text { and }\|u\|_{A_{p}(G)} \leq 1\right\}
$$

Observe that if $v \in A_{p}(G)$, then $v \in B_{p}(G)$ and $\|v\|_{B_{p}(G)} \leq\|v\|_{A_{p}(G)} \cdot B(G)$ denotes the Fourier-Stieltjes algebra of $G$. Then $B(G)=C^{*}(G)^{*}$ is the linear span of the continuous positive definite functions on $G$.

$G$ is said to be amenable if there exists $m \in L_{\infty}(G)^{*}$ such that $m \geq 0$, $m\left(1_{G}\right)=1$, and $m\left({ }_{x} f\right)=m(f)$ for every $x \in G, f \in L_{\infty}(G) .1_{A}$ denotes the characteristic function of $A$ and ${ }_{x} f(y)=f(x y)$. Amenable groups include all abelian groups and all compact groups. The free group on two generators $F_{2}$ is nonamenable.

A locally compact group is called a [SIN]-group if every neighborhood of $e$ contains a compact neighborhood which is invariant under all inner automorphisms. For properties of [SIN]-groups see [22].

Set $\mathscr{A}$ be a commutative Banach algebra. $\Delta(\mathscr{A})$ will denote both the maximal ideal space of $\mathscr{A}$ and the multiplicative linear functions associated with these ideals. By means of the Gelfand transform, $\mathscr{A}$ can be realized as a subalgebra of $C_{0}(\Delta(\mathscr{A}))$. For an ideal $I$ in $\mathscr{A}$, we define

$$
Z(I)=\{x \in \Delta(\mathscr{A}) \mid u(x)=0 \text { for every } u \in I\} .
$$

For $E \subset \Delta(\mathscr{A})$, define

$$
\begin{aligned}
I(E) & =\{u \in \mathscr{A} \mid u(x)=0 \text { for every } x \in E\} \\
I_{0}(E) & =\{u \in \mathscr{A} \mid \operatorname{supp} u \in \mathscr{F}(E)\}
\end{aligned}
$$

where $\mathscr{F}(E)=\{K \subset \Delta(\mathscr{A}) \mid K$ is compact and $K \cap \bar{E}=\varnothing\} . I(E)$ and $I_{0}(E)$ are ideals in $\mathscr{A} . I(E)$ is closed. Moreover, if $Z(I)=E$, then $I_{0}(E) \subseteq I \subseteq$ $I(E)$.

A closed subset $E$ of $\Delta(\mathscr{A})$ is called a set of spectral synthesis, or simply an $s$-set if $I(E)$ is the only closed ideal $I$ for which $Z(I)=E$. 
Let $G$ be a locally compact group. Let $A, B \subset G$ be closed. Let

$$
\begin{aligned}
& \mathscr{S}_{p}(A, B)=\left\{u \in B_{p}(G) \mid u(A) \equiv 1, u(B) \equiv 0\right\}, \\
& s_{p}(A, B)= \begin{cases}\inf \left\{\|u\|_{B_{p}(G)} \mid u \in \mathscr{S}_{p}(A, B)\right\} & \text { if } \mathscr{S}_{p}(A, B) \neq \varnothing, \\
\infty & \text { if } \mathscr{S}_{p}(A, B)=0,\end{cases} \\
& \mathscr{F}(A)=\{K \subset G \mid K \text { is compact, } K \cap A=\varnothing\}, \\
& s_{p}(A)=\sup \left\{s_{p}(A, K) \mid K \in \mathscr{F}(A)\right\}, \\
& I_{p}(A)=\left\{u \in A_{p}(G) \mid u(x)=0 \text { for every } x \in A\right\} .
\end{aligned}
$$

A net $\left\{u_{\alpha}\right\}_{\alpha \in \mathscr{U}}$ in $A_{p}(G)$ is a bounded approximate identity in an ideal $I$ if $u_{\alpha} \in I,\left\|u_{\alpha}\right\|_{A_{p}(G)} \leq M$ for all $\alpha \in \mathscr{U}$, and $\lim _{\alpha}\left\|u u_{\alpha}-u\right\|_{A_{p}(G)}=0$ for every $u \in I$. Since left and right translations are isometric isomorphisms, the ideals $I(A), I(x A)$, and $I(A x)$ are all isometric ismorphic.

An ideal $I$ is said to be idempotent if $I=I^{2}$, where $I^{2}=\left\{\sum_{i=1}^{n} u_{i} v_{i} \mid u_{i}\right.$, $\left.v_{i} \in I\right\}$.

\section{AMENABILITY, BOUNDED APPROXIMATE IDENTITIES AND WEAK FACTORIZATION}

In this section we will extend and improve many of the results of [9] and [10] to the setting of the $A_{p}(G)$ algebras, $1<p<\infty$. We will also consider weak factorization of ideals in $A_{p}(G)$.

Lemma 3.1. Let $G$ be an amenable locally compact group. Let $u \in B(G)$. Then $u \in B_{p}(G)$ for every $1<p<\infty$. Furthermore, for each $I<p<\infty$, there exists a constant $C_{p}$ independent of $u$ such that $\|u\|_{B_{p}(G)} \leq C_{p}\|u\|_{B(G)}$.

Proof. Since $G$ is amenable, $B_{p}(G)=\operatorname{PF}_{p}(G)^{*}$. Let $K \subset G$ be compact. Since $u \in B(G)$, Cowling [3] has shown that $u_{\left.\right|_{K}} \in A_{2}(K)=\left\{v_{\left.\right|_{K}} ; v \in A(G)\right\}$ and that

$$
\left\|u_{\left.\right|_{K}}\right\|_{A_{2}(K)}=\inf \left\{\|v\|_{A(G)} ; v_{\left.\right|_{K}}=u_{\left.\right|_{K}}\right\} \leq\|u\|_{B(G)} .
$$

Let $i: A(G) \rightarrow A_{p}(G)$ be the canonical injection. Since $i$ is continuous [15], there exists a constant $C_{p}$ such that $\|v\|_{A_{p}(G) \leq C_{p}}\|v\|_{A(G)}$ for every $v \in A(G)$. Let $v_{0} \in A(G)$ be such that $v_{\left.0\right|_{K}}=u_{\left.\right|_{K}}$. Then $i\left(v_{0}\right)_{\left.\right|_{K}}=u_{\left.\right|_{K}}$, so $u_{\left.\right|_{K}} \in A_{p}(K)$. Moreover,

$$
\begin{aligned}
\left\|u_{\left.\right|_{K}}\right\|_{A_{p}(K)} & \leq \inf \left\{\|i(v)\|_{A_{p}(G)} ; v \in A(G), v_{\left.\right|_{K}}=u_{\left.\right|_{K}}\right\} \\
& \leq \inf \left\{C_{p}\|v\|_{A_{p}(G)} ; v \in A(G), v_{\left.\right|_{K}}=u_{\left.\right|_{K}}\right\} \\
& \leq C_{p}\|u\|_{B(G)} .
\end{aligned}
$$

By the converse of Cowling's result, $u \in B_{p}(G)$ and $\|u\|_{B_{p}(G)} \leq C_{p}\|u\|_{B(G)}$.

Proposition 3.2. Let $G$ be an amenable locally compact group. Let $A \subset G$ be closed. If $s_{2}(A)<\infty$, then $s_{p}(A)<\infty$ for every $1<p<\infty$.

Proof. It follows from Lemma 3.1 that if $K \in \mathscr{F}(A)$ and $u \in \mathscr{I}_{2}(A, K)$, then $u \in \mathscr{S}_{p}(A, K)$ and $\|u\|_{B_{p}(G) \leq C_{p}}\|u\|_{B(G)}$. Hence, $s_{p}(A) \leq C_{p} s_{2}(A)$.

Corollary 3.3. Let $G$ be an amenable locally compact group. Let $H$ be a closed subgroup of $G$ which is either (i) open, (ii) compact, (iii) normal. Then $s_{p}(H)<$ $\infty$. Furthermore, if $G$ is $a$ [SIN]-group and $H$ is any closed subgroup, then $s_{p}(H)<\infty$.

Proof. If $H$ satisfies (i), (ii), or (iii) above, then by [10, Lemma 3.6], $s_{2}(H)=$ 1. Proposition 3.2 implies that $s_{p}(H)<\infty$. 
If $G$ is a [SIN]-group, then $s_{2}(H)=1$ for every closed subgroup $H$ of $G$ [11, Proposition 3.10]. Again, by Proposition 3.2, $s_{p}(H)<\infty$.

A straightforward modification of the proof of [10, Proposition 3.2] establishes the next proposition.

Proposition 3.4. Let $G$ be an amenable locally compact group. Let $A$ be a closed set of spectral synthesis for $A_{p}(G)$. If $s_{p}(G)<\infty$, then $I_{p}(A)$ has a bounded approximate identity $\left\{u_{\alpha}\right\}_{\alpha \in \mathscr{U}}$ which satisfies

(i) $\left\|u_{\alpha}\right\|_{A_{p}(G)} \leq 2+s_{p}(A)$ for every $\alpha \in \mathscr{U}$,

(ii) $u_{\alpha} \in A_{p}(G) \cap C_{00}(G)$ for every $\alpha \in \mathscr{U}$,

(iii) if $K \in \mathscr{F}(A)$, there exists a sequence $\left\{u_{K_{n}}\right\} \subseteq\left\{u_{\alpha}\right\}_{\alpha \in \mathscr{C}}$ such that $\left\|v u_{K_{n}}-v\right\|_{A_{p}(G)} \leq 1 / n$ for every $v \in A_{p}(G)$ with $\operatorname{supp} v \subseteq K$.

Corollary 3.5. Let $G$ be an amenable locally compact group. Let $H$ be a closed subgroup of $G$ which is (i) open, (ii) compact, or (iii) normal. Then $I_{p}(H)$ has a bounded approximate identity for every $1<p<\infty$ and hence $I_{p}(H)$ is idempotent. If $G$ is a [SIN]-group, then $I_{p}(H)$ has a bounded approximate identity, and hence is idempotent for every closed subgroup $H$ of $G$ and every $1<p<\infty$.

Proof. Since $G$ is amenable, every closed subgroup is a set of spectral synthesis for each $A_{p}(G), 1<p<\infty$ [14]. The result follows immediately from Corollary 3.5 and from Cohen's Factorization Theorem [17, Theorem 32.22].

Definition 3.6. Let $\mathscr{R}(G)$ denote the ring of subsets of $G$ generated by the open left-cosets of $G$. Define $\mathscr{R}_{c}(G)=\left\{A \subset G ; A\right.$ is closed, $\left.A \in \mathscr{R}_{(}\left(G_{d}\right)\right\}$ where $G_{d}$ is the group $G$ together with the discrete topology. The sets $A \in \mathscr{R}_{c}(G)$ can be characterized by following an argument due to Gilbert [12] originally presented for abelian groups. We have $A \in \mathscr{R}_{c}(G)$ if and only if $A=\bigcup_{i=1}^{n} x_{i}\left(H_{i} \backslash \Delta_{i}\right)$ where $H_{i}$ is a closed subgroup of $G, \Delta_{i} \in \mathscr{R}\left(H_{i}\right)$, and $x_{i} \in G$ for every $1 \leq i \leq n$ [11, Lemma 3.5].

Theorem 3.7. Let $G$ be an amenable $[\mathrm{SIN}]$-group. Let $A \in \mathscr{R}_{c}(G)$. Then $I_{p}(A)$ has a bounded approximate identity $\left\{u_{\alpha}\right\}_{\alpha \in \mathscr{U}}$ such that

(i) $u_{\alpha} \in A_{p}(G) \cap C_{00}(G)$,

(ii) if $K \in \mathscr{F}(A)$, then there exists a sequence $\left\{u_{K_{n}}\right\} \subseteq\left\{u_{\alpha}\right\}_{\alpha \in \mathscr{U}}$ such that if $v \in I(A)$ and $\operatorname{supp} v \subseteq K$, then

$$
\left\|u_{K_{n}} v-v\right\|_{A_{p}(G)} \leq 1 / n .
$$

In particular, $A$ is an s-set for $A_{p}(G)$.

Proof. That $A$ is an $s$-set follows immediately if we establish the existence of a bounded approximate identity satisfying (i).

It follows from Corollary 3.5 that $I_{p}(H)$ has a bounded approximate identity for any closed subgroup $H$ of $G$.

Let $A=\bigcup_{i=1}^{n} x_{i}\left(H_{i} \backslash \Delta_{i}\right)$ where $\Delta_{i} \in \mathscr{R}\left(H_{i}\right)$. By Host's idempotent theorem, $1_{\Delta_{i}} \in B(H i)$. By [4, Theorem 2], $1_{\Delta_{i}}$ extends to a $u \in \mathscr{S}_{2}\left(\Delta_{i}, H_{i} \backslash \Delta_{i}\right)$. Lemma 3.1 shows that $u \in \mathscr{S}_{p}\left(\Delta_{i}, H_{i} \backslash \Delta_{i}\right)$.

As $G$ is amenable, $A_{p}(G)$ has a bounded approximate identity [24, p. 96]. Arguing as in [11, Lemma 3.9 and Theorem 3.11], we see that $I_{p}\left(H_{i}\right)$ has a bounded approximate identity and therefore so must $I_{p}\left(H_{i} \backslash \Delta_{i}\right)$. It is then clear that $I_{p}\left(x_{i}\left(H_{i} \backslash \Delta_{i}\right)\right)$ has a bounded approximate identity. By again following 
[11, Theorem 3.11], we see that $I_{p}(A)$ has a bounded approximate identity $\left\{u_{\alpha}\right\}_{\alpha \in \mathscr{U}}$.

That $\left\{u_{\alpha}\right\}_{\alpha \in \mathscr{U}}$ can be chosen to satisfy (i) and (ii) follows from a careful examination of the proofs of [10, Proposition 3.2; 11, Lemma 3.9, and Theorem 3.11].

Theorem 3.8. Let $G$ be an amenable group. Let $X$ be a weak*-closed $A_{p}(G)$ submodule of $\operatorname{PM}_{p}(G)$. Then the following are equivalent:

(i) $X$ is invariantly complemented,

(ii) ${ }^{\perp} X$ has a bounded approximate identity.

Furthermore, if $G$ is any locally compact group for which ${ }^{\perp} X$ has a bounded approximate identity whenever $X$ is a weak*-closed invariantly complemented submodule of $\operatorname{PM}_{p}(G)$, then $G$ is amenable.

Proof. As $G$ is amenable, $A_{p}(G)$ has a bounded approximate identity. The first part follows from [10, Proposition 6.4].

Let $X=\{0\}$. Then $X$ is invariantly complemented and $A_{p}(G)={ }^{\perp} X$ has a bounded approximate identity if and only if $G$ is amenable so the last statement follows.

Corollary 3.9. Let $G$ be an amenable locally compact group. Let $H$ be a closed subgroup of $G$ which is (i) open, (ii) compact or (iii) normal. Then $I_{p}(H)^{\perp}$ is invariantly complemented. Furthermore, if $G$ is also $a$ [SIN]-group and $A \in$ $\mathscr{R}_{c}(G)$, then $I_{p}(A)^{\perp}$ is invariantly complemented.

Corollary 3.9 extends for the class of amenable groups a recent result of Derighetti [6, Théorème 2] who shows that $I_{p}(H)^{\perp}$ is invariantly complemented whenever $H$ is a closed normal subgroup and $G$ is an arbitrary locally compact group. In the case $p=2$, Derighetti's result is due to Lau and Losert [21, Theorem 2].

Theorem 3.10. Let $G$ be an abelian locally compact group. Then for every $1<$ $p<\infty, A_{p}(G)$ is an amenable Banach algebra.

Proof. Let $\widehat{G}$ denote the dual group of $G$. Then since $\widehat{G}$ is amenable, $L^{1}(\widehat{G})$ is amenable [19, Theorem 2.5]. Hence $A(G)$ is an amenable Banach algebra. For every $1<p<\infty, A(G)$ embeds continuously into $A_{p}(G)$ as a dense subalgebra. Therefore, $A_{p}(G)$ is amenable [19, Proposition 5.3].

Corollary 3.11. Let $G$ be an abelian locally compact group. Let $X$ be a weak*closed $A_{p}(G)$-submodule of $\operatorname{PM}_{p}(G)$. Then $X$ is complemented if and only if $X$ is invariantly complemented.

Proof. Assume that $X$ is complemented. Then since $A_{p}(G)$ is amenable, ${ }^{\perp} X$ has a bounded approximate identity [5, Theorem 3.7]. It follows from Theorem 3.8 that $X$ is invariantly complemented.

The case $p=2$ in Corollary 3.11 is again due to Lau and Losert [21]. It is worthwhile to note that Theorem 3.10 and Corollary 3.11 will hold whenever $G$ is such that $A(G)$ is amenable. There is reason to believe that this is the case precisely when $G$ is amenable.

For $A \subseteq G$, let bdy $A$ and int $A$ denote the boundary of $A$ and the interior of $A$ respectively. 
Proposition 3.12. Let $A \subset G$ be closed. Assume that $I_{p}(A)$ factorizes weakly. Then $\lambda(\operatorname{bdy} A)=0$ and $\operatorname{int}(A)$ is a clopen subset of $G$.

Proof. $I_{p}(A)$ is a weakly selfadjoint subalgebra of $A_{p}(G)$. Hence by [8, Theorem 1.3], there exists an $0<M<\infty$ such that for every $K \in \mathscr{F}(A)$, there exists a $u_{K} \in I_{p}(A)$ with $\left\|u_{K}\right\|_{A_{p}(G)} \leq M, u_{K}(x) \geq 1$ for every $x \in K$ and $u_{K} \geq 0$. Let $\mathscr{F}(A)$ be directed by inclusion. Then $\left\{u_{K}\right\}_{K \in \mathscr{F}(A)}$ is a bounded net in $W_{p}(G)=\mathrm{PF}_{p}(G)^{*}$. Hence we can assume that $\left\{u_{K}\right\}_{K \in \mathscr{F}(A)}$ converges in the weak *-topology to some $u \in W_{p}(G)$ with $\|u\|_{W_{p}(G)} \leq M$ (otherwise choose a convergent subnet). It is easy to see that $u(x) \geq 0, u(x) \geq 1$ on $G \backslash A$ and that $u(x)=0$ for every $x \in \operatorname{int}(A)$. Hence $u(x) \geq 1$ on $\operatorname{bdy}(A)$. Since $u$ is continuous, $\operatorname{int}(A)$ is clopen.

Assume that $\lambda(\operatorname{bdy} A)>0$. Then we can find a subset $V$ of bdy $A$ with $0<\lambda(V)<\infty$. Let $f=1_{V}$. Then $f \in L_{1}(G) \subseteq \operatorname{PF}_{p}(G)$ and

$$
0<\lambda(V) \leq \int_{G} u(x) f(x) d x=\langle u, f\rangle=\lim _{k}\left\langle u_{K}, f\right\rangle=0
$$

since $u_{K}(x)=0$ for every $x \in \operatorname{bdy} A$. As this is impossible, $\lambda(\operatorname{bdy} A)=0$.

Proposition 3.13. Let $A \subset G$ be closed. Suppose that $I$ is a closed ideal of $A_{p}(G)$ with $Z(I)=A$. If $I$ has a bounded approximate identity, then $\lambda(\operatorname{bdy} A)=0$ and $\operatorname{int}(A)$ is clopen. Moreover, $1_{G \backslash \operatorname{int} A} \in W_{p}(G)=\operatorname{PF}_{p}(G)^{*}$.

Proof. Let $\left\{u_{\alpha}\right\}_{\alpha \in \mathscr{U}}$ be a bounded approximate identity in $I$. We may assume that $\mathrm{w}^{*}-\lim _{\alpha} u_{\alpha}=u$ for some $u \in W_{p}(G)$.

Let $v \in I$. Let $f \in L_{1}(G)$ and $\varepsilon>0$. Then

$$
\langle u v, f\rangle=\langle u, v f\rangle=\lim _{\alpha}\left\langle u_{\alpha}, v f\right\rangle=\lim _{\alpha}\left\langle u_{\alpha} v, f\right\rangle \text {. }
$$

Let

$$
(*)=|\langle u v, f\rangle-\langle v, f\rangle| \leq\left|\langle u v, f\rangle-\left\langle u_{\alpha} v, f\right\rangle\right|+\left|\left\langle u_{\alpha} v, f\right\rangle-\langle v, f\rangle\right| .
$$

Choose $\alpha$ such that $\left|\langle u v, f\rangle-\left\langle u_{\alpha} v, f\right\rangle\right|<\varepsilon$ and $\left\|u_{\alpha} v-v\right\|_{A_{p}(G)}<\varepsilon$. Then $(*) \leq \varepsilon+\varepsilon\|f\|_{\mathrm{PF}_{p}(G)}$. Since $\varepsilon$ is arbitrary and $f$ is fixed, it follows that $\langle u v, f\rangle=\langle v, f\rangle$ for every $f \in L_{1}(G)$. Hence $u v=v$. It follows also that $u(x)=1$ on $G \backslash A$ and that $u(x)=0$ on int $A$. Therefore $u=1_{G \backslash \operatorname{int} A}$.

Proposition 3.14. Let $I$ be a closed ideal in $A_{p}(G)$ with $Z(I)=A$. If either $I$ is weakly selfadjoint or $I$ has a bounded approximate identity, then either $\lambda(A)>0$ or $G$ is amenable.

Proof. Assume that $\lambda(A)=0$. Let $K \subset G$ be compact. Let $y \in C_{00}^{+}(G)$, with $\operatorname{supp} \varphi \subseteq K$. Let $\varepsilon, \varepsilon_{1}>0$. We can find an open neighborhood $V_{\varepsilon_{1}}$ of $A$ such that $\lambda(V)\|\varphi\|_{\infty}<\varepsilon_{1}$.

In either of the above cases, we can find a $u \in I$ with $\|u\|_{A_{p}(G)}<M<\infty$ ( $M$ independent of $K$ ) which is such that $\inf \left\{R_{e} u(x) ; x \in K \backslash V\right\} \geq 1-\varepsilon$. Then

$$
|\langle u, \varphi\rangle| \leq\left\|L_{\varphi}\right\|_{C V_{p^{\prime}}}\|u\|_{A_{p}(G)} \leq M\left\|L_{\varphi}\right\|_{C V_{p^{\prime}}}
$$

where $\left\|L_{\varphi}\right\|_{C V_{p^{\prime}}}$ is the norm of $\varphi$ as a convolution operator on $L_{p^{\prime}}(G)$.

But

$$
R_{e}\langle u, \varphi\rangle=\int_{G} R_{e} u(x) \varphi(x) d x \geq(1-\varepsilon)\|\varphi\|_{1}-\varepsilon_{1} .
$$

Therefore $\|\varphi\|_{1} \leq M\left\|L_{\varphi}\right\|_{C V_{p^{\prime}}}$. 
As $K$ was arbitrary, $\|\psi\|_{1} \leq M\left\|L_{\psi}\right\|_{C V_{p^{\prime}}}$ for every $\psi \in C_{00}^{+}(G)$. Given $\psi \in C_{00}^{+}(G)$, we have

$$
\|\psi\|_{1}^{n}=\left\|\psi^{* n}\right\|_{1} \leq M\left\|L_{\psi^{* n}}\right\|_{C V_{p^{\prime}}} \leq M\left\|L_{\psi}\right\|_{C V_{p^{\prime}}} .
$$

Hence $\|\psi\|_{1}=\left\|L_{\psi}\right\|_{C V_{p^{\prime}}}$ for every $\psi \in C_{00}^{+}(G)$. This implies that $G$ is amenable.

In [11], we established a connection between the existence of bounded approximate identities in $A(G)$ and the existence of either "large" amenable subgroups or open amenable subgroups. It was conjectured that if there is a closed ideal in $A(G)$ with a bounded approximate identity, then $G$ must have an open amenable subgroup. We have further evidence to support this conjecture and more.

Theorem 3.15. Let $G$ be a connected locally compact group. Let $I$ be a nonzero closed ideal in $A_{p}(G), 1<p<\infty$, which is such that either I has a bounded approximate identity or $I$ is weakly selfadjoint and weakly factorizes. Then $G$ is amenable.

Proof. Let $Z(I)=A$. If $G$ is nonamenable, then by Proposition $3.14 \lambda(A)>$ 0 . It follows from Propositions 3.12 and 3.13 that $\operatorname{int}(A)$ is a nonempty clopen set. Since $G$ is connected, $A=G$. But then $I=\{0\}$ which is impossible. Hence $G$ is amenable.

Corollary 3.16. Let $G$ be an almost connected locally compact group. Let $I$ be a nonzero closed ideal in $A_{p}(G), 1<p<\infty$, with a bounded approximate identity, then $G$ is amenable.

Proof. Let $A=Z(I)$. Since $I$ is nonzero, $A \neq G$. Therefore, by translating if necessary, we can assume that there exist $x_{0} \in G_{0} \backslash A$, where $G_{0}$ is the connected component of $G$. Let $I_{G_{0}}=\left\{u_{\mid G_{0}} ; u \in I\right\}^{-}$(the closure of $\left\{u_{\mid G_{0}} ; u \in I\right\}$ in $\left.A_{p}(G)\right)$. Since $A_{p}(G)_{\mid G_{0}}=A_{p}\left(G_{0}\right)$ [16, Theorem 1], $I_{G_{0}}$ is a closed ideal in $A_{p}\left(G_{0}\right)$. Let $\left\{u_{\alpha}\right\}_{\alpha \in \mathscr{U}}$ be a bounded approximate identity in $I$. As $\left\|u_{\mid G_{0}}\right\|_{A_{p}\left(G_{0}\right)} \leq\|u\|_{A_{p}(G)}$ for every $u \in A_{p}(G),\left\{u_{\mid G_{0}}\right\}_{\alpha \in \mathscr{U}}$ is a bounded approximate identity for the closed ideal $I_{G_{0}}$, which is nonzero since $x_{0} \in G_{0} \backslash A$. By Theorem 3.15, $G_{0}$ is amenable. But $G$ is almost connected, so $G / G_{0}$ is compact and hence amenable. Therefore $G$ is also amenable [24, Proposition 13.4].

\section{Cofinite ideals in $A_{p}(G)$}

Definition 4.1. An ideal $I$ in $A_{p}(G)$ is called cofinite if the dimension of $A_{p}(G) / I$ is finite. The codimension of $I$ is $\operatorname{dim} A_{p}(G) / I$.

We can proceed as in $[10, \S 5]$ to obtain the following characterization of amenable groups which extends [10, Corollary 5.6, Lemma 5.7, and Theorem 5.8].

Theorem 4.2. Let $G$ be a locally compact group. Then the following are equivalent:

(a) $G$ is amenable.

(b) For every $1<p<\infty$ and every cofinite ideal $I \subset A_{p}(G), I=I(A)$ for some finite set $A=\left\{x_{1}, \ldots, x_{n}\right\} \subset G$ with $n=\operatorname{codim} I$. 
(c) For every $1<p<\infty$ and every cofinite ideal $I \subset A_{p}(G)$, I has a bounded approximate identity.

(d) For some $1<p<\infty$ and some closed cofinite ideal $I \subset A_{p}(G), I^{2}=I$.

Lemma 4.3. Let $G$ be an amenable locally compact group. Let $I$ be a closed in $A_{p}(G)$ with infinite codimension. Then there exist sequences $\left\{u_{n}\right\},\left\{v_{n}\right\}$ in $A_{p}(G)$ such that $u_{n} v_{1} \cdots v_{n-1} \notin I$ but $u_{n} v_{1} \cdots v_{n} \in I$. 2]).

The proof of this lemma is identical to that of the case $p=2$ (see [9, Lemma

Definition 4.4. Let $\mathscr{A}$ be a Banach algebra and let $X$ be a Banach $\mathscr{A}$-bimodule. A derivation $D: \mathscr{A} \rightarrow X$ is a linear map which satisfies $D(u v)=u \cdot D(v)+$ $D(u) \cdot v$ for every $u, v \in \mathscr{A}$. Let $X$ be a left Banach $\mathscr{A}$-module. A linear operator $T: \mathscr{A} \rightarrow X$ is said to be of class $\mathscr{T}$ if for every $u, v \in \mathscr{A}$

$$
T(u v)=u \cdot T(v)+L(u, v)
$$

where $L(\cdot, \cdot)$ is a bilinear operator from $\mathscr{A} \times \mathscr{A}$ to $X$ for which $v \mapsto L(u, v)$ is continuous for each $u \in \mathscr{A}$.

Proceeding as in [9, Theorem 1] and [10, Theorem 5.9] we have the following

Theorem 4.5. Let $G$ be a locally compact group. Then the following are equivalent:

(i) $G$ is amenable.

(ii) For every $1<p<\infty$, every homomorphism from $A_{p}(G)$ with finitedimensional range is continuous.

(iii) For every $1<p<\infty$, every derivation of $A_{p}(G)$ into a finite-dimensional commutative Banach $A_{p}(G)$-bimodule is continuous.

(iv) For every $1<p<\infty$, every derivation of $A_{p}(G)$ into a Banach $A_{p}(G)$ bimodule is continuous.

Theorem 4.6. Let $G$ be a compact group. Let $1<p<\infty$. If $S: A_{p}(G) \rightarrow X$ is of class $\mathscr{g}$, then $S$ is continuous.

Proof. First assume that $G$ is separable. Then $A_{p}(G)$ is a separable Banach algebra with identity. Since $A_{p}(G)$ is a normal algebra, every prime ideal is contained in a unique maximal ideal (see [1, p. 97]). Let $J$ be a closed prime ideal. Then since $Z(J)=\left\{x_{0}\right\}$ for some $x_{0} \in G, J=I\left(\left\{x_{0}\right\}\right)$. Hence $J$ is a maximal ideal. Moreover, if $I=I(\{x\})$ is a maximal ideal, then $I^{2}=I$. It follows from [1, Theorem 4.2] that every operator of class $\mathscr{F}$ is bounded.

Now assume that $G$ is an arbitrary compact group and that $T: A_{p}(G) \rightarrow X$ is class $\mathscr{F}$. Assume that $T$ is discontinuous. Let $\left\{u_{n}\right\}$ be a sequence in $A_{p}(G)$ such that $u_{n} \rightarrow 0$ while $T u_{n} \nrightarrow 0$. For each $n$ we can find a compact normal subgroup $K_{n}$ of $G$ such that $G / K_{n}$ is separable and $u_{n}$ is constant on cosets of $K_{n}$. Let $K=\bigcap_{n=1}^{\infty} K_{n}$. Then $K$ is compact and normal. Furthermore each $u_{n}$ is constant on cosets of $K$ and $G / K$ is separable.

There exists an isometric isomorphism $\rho$ from $A_{p}(G / K)$ onto the subspace of $A_{p}(G)$ consisting of functions which are constant on cosets of $K$ [16, Proposition 6]. $X$ becomes a left Banach $A_{p}(G / K)$-module with respect to the module action as defined by $\tilde{u} \circ x=\rho(\tilde{u}) x$ for every $\tilde{u} \in A_{p}(G / K)$. Similarly $\widetilde{T}: A_{p}(G / K) \rightarrow X$ defined by $\widetilde{T}(\tilde{u})=T(\rho(\tilde{u}))$ for every $\tilde{u} \in A_{p}(G / K)$ is of 
class $\mathscr{F}$. If we choose $\tilde{u}_{n} \in A_{p}(G / K)$ such that $\rho\left(\tilde{u}_{n}\right)=u_{n}$, then $\tilde{u}_{n} \rightarrow 0$, but $\widetilde{T}\left(\tilde{u}_{n}\right) \nrightarrow 0$. Hence $\widetilde{T}$ is discontinuous. By the above argument, this is impossible.

Remark. The class $\mathscr{T}$ was introduced by Bade and Curtis [1] in order to handle both homomorphisms and derivations simultaneously in their investigation of automatically continuous linear functions. They also considered the linear map $T$ from $\mathscr{A}$ into a Banach $\mathscr{A}$-bimodule which satisfies Leibniz rule of order $n$. That is, there exist operators $T_{j}$ and $\widetilde{T}_{j}, j=1, \ldots, n-1$, such that $T_{j}$ and $\widetilde{T}_{j}$ satisfy a Leibniz rule of order $j$ and

$$
T(a b)=a T(b)+\sum_{j=1}^{n-1} T_{j}(a) \widetilde{T}_{n-j}(b)+T(a) b .
$$

In particular, if $T_{j}=\widetilde{T}_{j}$, then $\left\{T_{1}, \ldots, T_{n}\right\}$ is called a higher derivation of rank $n$. It is a simple induction argument to show that if $S$ satisfies a Leibniz rule of order $n$ then $S$ is bounded if every $T: \mathscr{A} \rightarrow X$ of class $\mathscr{T}$ is bounded.

Bade and Curtis also observe that if $X$ is a separable Banach space with an ordered Schauder basis $\left\{x_{i}\right\}$ and if $\mathscr{A}$ is an algebra such that every operator that satisfies a Leibniz rule of order $n$ is continuous, then every homomorphism $\rho$ from $\mathscr{A}$ into $\mathscr{B}(X)$ for which $\rho(u)$ is upper triangular for all $u \in \mathscr{A}$ is continuous (see [1, pp. 99-100]).

Theorem 4.7. Let $G$ be a compact group. Let $1<p<\infty$. Let $X$ be a Banach $A_{p}(G)$-bimodule. If $T: A_{p}(G) \rightarrow X$ is a linear map which satisfies a Liebniz rule of order $n$, then $T$ is continuous. In particular, if $\left\{T_{1}, \ldots, T_{n}\right\}$ is a derivation of rank $n$ on $A_{p}(G)$, then each $T_{i}$ is continuous.

Corollary 4.8. Let $G$ be a compact group. Let $1<p<\infty$. Let $X$ be a Banach space with an ordered Schauder basis. If $\rho: A_{p}(G) \rightarrow \mathscr{B}(X)$ is a homomorphism for which each $\rho(u), u \in A_{p}(G)$, can be represented by an upper triangular matrix, then $\rho$ is continuous.

In [26], Warner and Whitley proved that if $X$ is a closed subspace in $L^{1}(\mathbb{R}) \cong$ $A(\mathbb{R})$ of codimension $n$ for which every $f \in X$ belongs to at least $n$ distinct maximal ideals, then $X$ is an ideal. In particular, $X \cong I\left(\left\{x_{1}, \ldots, x_{n}\right\}\right)$ for some subset $\left\{x_{1}, \ldots, x_{n}\right\}$ of $\mathbb{R}$. This result is related to a classic theorem of Gleason-Kahane-Zelazko for subspaces of codimension 1 in unitary Banach algebras (see $[13,20])$. Warner and Whitley ask for which locally compact abelian groups does $L^{1}(G)$ and hence $A(G)$ have this property. Recent work of Chen and Cohen [2] has led to the solution of this question. Using a recent result of Rao, we may prove the following.

Proposition 4.9. Let $G$ be a locally compact group. Let $1<p<\infty$. Assume that $g$ is $\sigma$-compact and separable. Then $A_{p}(G)$ has property $(*)$ :

(*) If $X$ is a closed subspace of $A_{p}(G)$ of codimension $n$ which is such that every $u \in X$ vanishes at least $n$ distinct points in $G$, then $X$ is an ideal in $A_{p}(G)$. In particular, $X=I\left(\left\{x_{1}, \ldots, x_{n}\right\}\right)$ for some $\left\{x_{1}, \ldots, x_{n}\right\} \subset G$.

Moreover, if $A_{p}(G)$ has property $(*)$, then $G$ is $\sigma$-compact and separable.

Proof. The algebras $A_{p}(G)$ are all semisimple, selfadjoint regular Banach algebras. If $G$ is separable, then $\{x\}$ is a $G_{\delta}$-set for each $x \in G$. Finally, since $G$ 
is $\sigma$-compact, the statement follows from [25, Theorem 2.3] and the fact that finite subsets of $G$ are $s$-sets for $A_{p}(G)$.

Conversely, if $G$ is not $\sigma$-compact, then since $A_{p}(G) \subset C_{p}(G)$, every function $u \in A_{p}(G)$ vanishes at infinitely many points of $G$. Hence (*) cannot hold.

If $G$ is not separable and $u\left(x_{0}\right)=0$ for some $u \in A_{p}(G)$ and $x_{0} \in G$, then there exists a compact normal subgroup $K$ of $G, K \neq\{e\}$, such that $u\left(x_{0} k\right)=0$ for every $k \in K$. Hence $A_{p}(G)$ is not strongly separating and thus (*) fails (see [25, p. 242]).

\section{REFERENCES}

1. W. G. Badé and P. C. Curtis, Prime ideals and automatic continuity problems for Banach algebras, J. Funct. Anal. 29 (1978), 88-103.

2. C. P. Chen and P. J. Cohen, Ideals of finite codimension in commutative Banach algebras, preprint.

3. M. Cowling, An application of the Littlewood-Paley theory in harmonic analysis, Math. Ann. 241 (1979), 83-86.

4. M. Cowling and P. Rodway, Restriction of certain function spaces to closed subgroups of locally compact groups, Pacific J. Math. 80 (1979), 91-104.

5. P. C. Curtis and R. J. Loy, The structure of amenable Banach algebras, J. London Math. Soc. 40 (1989), 89-104.

6. A. Derighetti, Convoluteurs et projecteurs, Harmonic Analysis, Lecture Notes in Math., vol 1359, Springer-Verlag, Berlin and New York, 1987.

7. P. Eymard, L'algèbre de Fourier d'un groupe localement compact, Bull. Soc. Math. France 92 (1964), 181-236.

8. H. G. Feichtinger, C. C. Graham, and E. H. Lakien, Nonfactorization in commutative, weakly self-adjoint Banach algebras, Pacific J. Math. 80 (1979), 117-125.

9. B. E. Forrest, Amenability and derivations of the Fourier algebra, Proc. Amer. Math. Soc. 104 (1988), 437-442.

10. 34 (1990), 1-25.

11. _ Amenability and ideals in $A(G)$, J. Austral. Math. Soc. (Ser. A) 53 (1992), 143-155.

12. J. E. Gilbert, On projections of $L_{\infty}(G)$ onto translation-invariant subspaces, Proc. London Math. Soc. 19 (1969), 69-88.

13. A. M. Gleason, A characterization of maximal ideals, J. Analyse Math. 19 (1967), 171-172.

14. C. Herz, Synthése spectrale pour les sous-groupes par rapport aux algèbres $A_{p}, \mathrm{C} . \mathrm{R}$. Acad. Sci. Paris Sér. I Math. 271 (1970), 316-318.

15. The theory of p-spaces with application to convolution operators, Trans. Amer. Math. Soc. 154 (1971), 69-82.

16. __ Harmonic synthesis for subgroups, Ann. Inst. Fourier (Grenoble) 23 (1973), 91-123.

17. E. Hewitt and K. A. Ross, Abstract harmonic analysis, vol. II, Springer-Verlag, Berlin and New York, 1970.

18. B. Host, Le théorème des idempotents dans $B(G)$, Bull. Soc. Math. France 114 (1986), 215-223.

19. B. E. Johnson, Cohomology in Banach algebras, Mem. Amer. Math. Soc. 127 (1972).

20. J. P. Kahane and W. Zelazko, A characterization of maximal ideals in commutative Banach algebras, Studia Math. 29 (1968), 339-343.

21. A. T. Lau and V. Losert, Weak* closed complemented invariant subspaces of $L_{\infty}(G)$ and amenable locally compact groups, Pacific J. Math. 123 (1986), 149-159.

22. T. W. Palmer, Classes of nonabelian, noncompact, locally compact groups, Rocky Mountain J. Math. 8 (1978), 683-741. 
23. A. L. T. Paterson, Amenability, Amer. Math. Soc., Providence, RI, 1988.

24. J. P. Pier, Amenable locally compact groups, Wiley, New York, 1984.

25. N. V. Rao, Closed ideals of finite codimension of regular self-adjoint Banach algebras, J. Funct. Anal. 82 (1989), 237-258.

26. C. R. Warner and R. Whitley, Ideals of finite codimension in $C[0,1]$ and $L^{1}(\mathbb{R})$, Proc. Amer. Math. Soc. 76 (1979), 263-267.

Department of Pure Mathematics, University of Waterloo, Waterloo, Ontario, CANADA N2L 3G1

E-mail address: beforrest@poppy . uwaterloo.ca 